RePortes de Tesis De Posgrado

\title{
Diseño de intervenciones basadas en IHR para el manejo de comportamientos problemáticos en adultos mayores con demencia
}

\author{
Dagoberto Cruz-Sandoval
}

Publicado: 21 Septiembre 2016

\begin{abstract}
Resumen
La revisión de literatura e investigaciones exitosas con robots de asistencia social (RAS), ha motivado nuestra hipótesis de que la interacción entre adultos mayores con demencia y un RAS puede ayudar a mejorar algunos comportamientos problemáticos. Mi investigación doctoral se enfoca en diseñar e implementar intervenciones basadas en interacción humano-robot (IHR) para apoyar el manejo de algunos de los más frecuentes comportamientos problemáticos en adultos mayores con demencia.
\end{abstract}

Palabras clave: Interacción Humano-Robot; adultos mayores con demencia; comportamientos problemáticos; robot de asistencia social.

\section{Introducción}

Además del deterioro cognitivo, los Adultos mayores con Demencia (ACD) frecuentemente presentan síntomas psicológicos y conductuales (SPCD). Estos síntomas son también denominados comportamientos problemáticos, síntomas conductuales y emocionales, o síntomas neuropsiquiátricos [1]. Los SPCD se definen como síntomas de trastornos de la percepción, del razonamiento, del estado de ánimo o del comportamiento que frecuentemente ocurren en pacientes con demencia. Se estima que alrededor de dos tercios de los AcD experimentan algún SPCD en su evolución. El 90\% de los pacientes de Alzheimer presentan este tipo de comportamientos problemáticos, lo que convierte los cuidados del AcD en una tarea compleja y desafiante [5]. Algunos de los efectos asociados a estos comportamientos problemáticos son [5]:

a. Alto grado de sufrimiento en el paciente y su cuidador;

b. Mayor discapacidad en el paciente, con mayor pérdida de autonomía personal;

c. Ingresos hospitalarios o institucionalización prematura en residencias geriátricas;

d. Aumento del costo de los cuidados; y

e. Pérdida significativa de la calidad de vida para el paciente y su

Cruz-Sandoval, D.

Departamento de Ciencias de la Computación CICESE

Ensenada, México

Email: dagoberto@cicese.edu.mx

\section{familiar o cuidador}

La integración social es un componente clave para tener un envejecimiento exitoso, es decir, mantener el bienestar físico y mental del adulto mayor [2]. La falta de conexiones sociales, la poca participación en actividades sociales y el distanciamiento social, predicen un mayor riesgo de deterioro cognitivo en los adultos mayores [10]. La interacción social, específicamente la percepción de una relación social interpersonal, tiene un impacto positivo en el bienestar físico y mental, además de reducir la probabilidad de depresión [8].

Existen nuevos campos de la robótica, en donde la meta no solo es proporcionar asistencia física, sino brindar estimulación a los humanos a través de la interacción con un robot. Entre los diferentes servicios de salud que deben de proporcionarse a los adultos mayores, la terapia de ejercicios físicos, la interacción social y la compañía se pueden abordar por medio de Robots de Asistencia Social (RAS). Un RAS es un sistema que emplea estrategias de interacción "hands-off", incluyendo comunicación auditiva, expresiones faciales y gestos comunicativos, para proporcionar la asistencia de acuerdo a un contexto de cuidado de salud determinado [3]. El diseño de RAS es un reto importante dentro de la Interacción Humano-Robot (IHR), debido a que enfatiza la interacción próxima con personas que pueden llegar a tener algún tipo de discapacidad. Los desafíos incluyen el apoyo a las interacciones sociales efectivas a través computo cognitivo y afectivo, incluyendo interacciones naturales como gestos y lenguaje natural [6].

Trabajos relacionados con en el uso de RAS como apoyo en el cuidado de adultos mayores, han explorado diversas formas de interacción. El robot Bandit es un robot humanoide que motiva el ejercicio y la actividad mental del adulto mayor, empleando estrategias de personalización y motivación [3]. PARO es un robot diseñado específicamente para propósitos de terapia, y cubre algunas de las percepciones y necesidades del adulto mayor, incluyendo la atención y compañía por largos periodos, estimulación positiva emocional y social, y al mismo tiempo proporcionar tiempo libre a los cuidadores para realizar otras actividades [7]. Brian 2.1 es un RAS expresivo y con forma humana, cuyas principales funciones es motivar y asistir durante la actividad de comer, además de estimular cognitivamente al adulto mayor por medio de un juego de cartas [4]. 


\section{Objetivos y enfoque de investigación}

Los resultados mostrados en investigaciones del uso de RAS como apoyo en el cuidado de adultos mayores, han motivado el objetivo de nuestra investigación: Diseñar, implementar y evaluar intervenciones basadas en interacción humano-robot por medio de robots de asistencia social, para el apoyo en el manejo de comportamientos problemáticos en adultos mayores con demencia.

Las siguientes preguntas de investigación son relevantes para nuestro trabajo:

- ¿Cómo puede ser aplicada la IHR en el dominio de las intervenciones no farmacológicas para el manejo de comportamientos problemáticos?

- ¿Qué comportamientos problemáticos pueden ser manejados por medio de intervenciones basadas en IHR?

- ¿Bajo qué estrategias de interacción debe comportarse un RAS para interactuar con un AcD?

Nuestro enfoque de investigación está basado en el método de investigación a través del diseño, con múltiples iteraciones en el diseño, prototipado y evaluación de interacciones para apoyar intervenciones para el manejo de comportamientos problemáticos. Sin embargo, antes de diseñar e implementar una intervención IHR, es necesario comprender las necesidades y oportunidades, así como los aspectos más importantes en la vida diaria de los AcD. La primera fase de la investigación emplea métodos cualitativos como entrevistas y observación contextual, para un entender mejor las necesidades de interacción y afectivas de los AcD.

Otro punto importante es una revisión iterativa y profunda de literatura. El objetivo de una primera revisión de literatura de sistemas RAS para el apoyo de los cuidados en los AcD es caracterizar el estado del arte en este campo de investigación.

\section{Fase actual de investigación}

En las primeras etapas de trabajo, estoy enfocado en obtener y analizar datos para entender las necesidades y oportunidades en el dominio del problema. Por esto, hemos realizado casos de estudio para evaluar la viabilidad de usar un sistema RAS para apoyar el manejo de los comportamientos problemáticos que experimenta el AcD. La información ha sido obtenida a través de entrevistas con especialistas en el cuidado del AcD, además de observaciones contextuales en residencias geriátricas. El análisis incluye sesiones de interpretación y codificación por medio de teoría fundamentada.

Por medio de cuatro diferentes perspectivas de expertos en áreas relacionadas con el cuidado del AcD -geriatría, gerontología, psicología, terapias no farmacológicas- hemos obtenido los primeros resultados acerca de las necesidades y oportunidades en los problemas relacionados con comportamientos problemáticos en AcD. Además, esta información fue complementada por medio del análisis de observaciones contextuales en dos residencias geriátricas.

Para nuestros primeros casos de estudio, algunos de los resultados más importantes fueron:

- Los comportamientos problemáticos más frecuentes en las casas geriátricas son la ansiedad, depresión y agresión.

- La estrategia principal para el manejo de estos comportamientos problemáticos es la interacción social.

- Dentro de las interacciones sociales, las charlas y conversaciones son la estrategia más utilizada y efectiva.
- Las intervenciones no farmacológicas (e.g. yoga, ejercicio físico, juegos y conversaciones en grupo) pueden tener un efecto de relajación y calma para los adultos mayores.

En base a estos primeros resultados y debido a que el uso de una interfaz por voz es el modo de comunicación más poderoso que tiene un RAS para comunicarse con sus usuarios [9], actualmente nos hemos enfocado en las conversaciones entre RAS y AcD para el manejo de comportamientos problemáticos. Planteando para esta fase de nuestra investigación la hipótesis de que un RAS capaz de establecer un diálogo personalizado puede proporcionar compañía, distracción y relajación al AcD, para brindar apoyo en el manejo de comportamientos problemáticos. Con el objetivo de sustentar esta hipótesis, la etapa actual de mi tesis doctoral se centra en el diseño e implementación de modelos de dialogo en un RAS, para generar intervenciones conversacionales como apoyo en el manejo de comportamientos problemáticos en $\mathrm{AcD}$.

\section{Contribuciones esperadas}

A través de la investigación, diseño y análisis de las actividades descritas, mi tesis doctoral contará con las siguientes contribuciones:

- Una intervención basada en IHR para apoyar el manejo de comportamientos problemáticos en AcD

- Nuevas formas de interacción para fomentar la interacción social entre un robot y un AcD.

- Modelos de diálogo para establecer conversaciones personalizadas entre un robot y un AcD.

- Información cualitativa y cuantitativa acerca de la relevancia clínica y la aceptación de intervenciones basadas en IHR por los AcD y los cuidadores.

\section{Expectativas del consorcio de posgrado}

Es una gran oportunidad para discutir y presentar mi investigación y recibir comentarios constructivos por parte de un grupo de investigadores experimentados y de estudiantes en un foro de gran envergadura. Espero beneficiarme de los interesantes puntos de vista, consejos y comentarios para mi proyecto de investigación. Debido a que mi investigación se encuentra en sus primeras fases, espero poder tener una gran retroalimentación e ideas acerca de cómo delimitar y mejorar mi investigación.

\section{Referencias}

[1] Burns, K. et al. 2012. Behaviour Management - A Guide to Good Practice.

[2] Cornwell, B. et al. 2008. The Social Connectedness of Older Adults: A National Profile*. American sociological review. 73, 2 (2008), 185-203.

[3] Fasola, J. and Mataric, M.J. 2012. Using Socially Assistive Human-Robot Interaction to Motivate Physical Exercise for Older Adults. Proceedings of the IEEE. 100, 8 (Aug. 2012), 2512-2526.

[4] McColl, D. et al. 2013. Brian 2.1: A Socially assistive robot for the elderly and cognitively impaired. IEEE Robotics and Automation Magazine. 20, 1 (Mar. 2013), 74-83.

[5] Sadowsky, C.H. and Galvin, J.E. 2012. Guidelines for the management of cognitive and behavioral problems in dementia. J Am Board Fam Med. 25, 3 (2012), 350-366.

[6] Salvine, P. et al. 2011. Benefits of Human - Robot Interaction [TC Spotlight]. IEEE Robotics \& Automation Magazine. 18, 4 (Dec. 2011), 98-99. 
[7] Shibata, T. 2012. Therapeutic Seal Robot as Biofeedback Medical Device: Qualitative and Quantitative Evaluations of Robot Therapy in Dementia Care. Proceedings of the IEEE. 100, 8 (Aug. 2012), 2527-2538.

[8] Stice, E. et al. 2004. Prospective relations between social support and depression: differential direction of effects for parent and peer support? Journal of abnormal psychology. 113, 1 (Feb. 2004), 155-9.

[9] Tapus, A. and Chetouani, M. 2010. ROBADOM: The Impact of a Domestic Robot on the Psychological and Cognitive
State of the Elderly with Mild Cognitive Impairment. Proceedings of the Second International Symposium on Quality of Life Technology Intelligent Systems for Better Living (Las Vegas, USA, 2010).

[10] Zunzunegui, M.-V. et al. 2003. Social Networks, Social Integration, and Social Engagement Determine Cognitive Decline in Community-Dwelling Spanish Older Adults. The Journals of Gerontology Series B: Psychological Sciences and Social Sciences. 58, 2 (Mar. 2003), S93-S100 